\title{
Heavy Metals and Chelation Therapy Haitao Wu
}

\section{Summary}

Increasing evidence indicates that heavy metal overload can cause or contribute to a variety of diseases and chronic illnesses. Chelation therapy has been widely accepted as effective treatment for heavy metal poisoning, accumulation of iron and copper in the body, etc. However, its applications in treatments for disorders such as diabetes, heart disease, neurodegenerative disease and cancer have long been mired in controversy; and exaggerated, poorly controlled chelation therapy treatments that have failed to show noticeable efficacy have also aroused skepticism. The vague recognition of the relation between heavy metal overload and disorders, the slow progress of chelation agent development, the intrinsic obstacles of the chemical, biological and clinical restrictions and the scarce involvement of pharmaceutical industries have constrained the advance of chelation therapy from moving forward to a new level. However, new chelation agent development and recent advances of technology such as MRI have significantly promoted the chelation therapy study. Promising results have been reported in a growing number. Chelation therapy is approaching a turning point that should lead to rapid progress and regain public confidence. The inauguration of the Journal of Heavy Metal and Chelation Therapy would provide an appropriate platform to promote the advance of chelation therapy.
National Heart, Lung, and Blood Institute, National Institutes of Health, USA

\section{Corresponding Author: Haitao Wu}

National Heart, Lung, and Blood Institute, National Institutes of Health, USA

”wuh3@nhlbi.nih.gov

Lecturer, Nursing Department, Alexandreio Educational Institute of Thessaloniki, Greece

Tel: $301-217-5763$

Citation: Haitao Wu. Heavy Metals and Chelation Therapy. J Heavy Met Toxicity Dis. 2016, 1:1.

Received: September 18, 2015; Accepted: September 23 2015; Published: September 30, 2015

\section{Editorial}

Normal physiological function requires various minerals, including trace amounts of heavy metals such as zinc, copper and iron, as essential elements to maintain the metabolism. Heavy metals enter the body via food, drinking water, and even breathing air, but excessive levels can induce disorders and diseases. The past hundred years of industrialization and technological advances have brought enormous benefits and enhanced living standards, but have also increased environmental pollution and its adverse effects of which heavy metal pollution is among the most hazardous. The situation is even more severe in some developing countries where the economy has been prioritized over environmental effects. For example, it was not until 2015 that the rapidly developing and heavily polluted country China began to place pollution control ahead of economic growth.

The adverse effects of heavy metal contamination on humans come not only from high existing levels in the body, but also from low-level, chronic exposure. Increasing evidence reveals that heavy metal overload can cause or contribute to a variety of diseases and some chronic illnesses including lower childhood I.Q., birth defects, Young's syndrome, Wilson's disease, depression, memory loss, seizures, neuropathy, fibromyalgia, infertility, and many other idiopathic illnesses that do not fit into traditional medical models. Heavy metal overexposure can be combated by chelation therapy, a medical treatment that removes metal toxins from the bloodstream by sequestering them in a chemically inert chelate form that can then be excreted in the urine.

Chelation therapy was initially developed as an antidote to heavy metal poisoning or radioactive contaminant exposure during WWII. Many standard pharmaceutical chelation agents have been developed since then to treat heavy metal toxicity. Some examples of chelation therapy are widely accepted as being effective, such as treatment of heavy metal poisoning, accumulation of iron in the body due to repeated blood transfusions, accumulation of copper due to Wilson's disease, overdose of medications containing metal ions and internal radioactive metal contamination. However, chelation therapy approaches to treating other disorders such as diabetes, heart disease, neurodegenerative disease, cancer, and autism have been mired in controversy. Poorly designed and controlled studies and chelation therapy practices in these areas have also incited 
many debates. Fundamental research into chelation therapy is not keeping pace with the demand and enthusiasm for the approach. Currently, only a few agents for chelation treatment have been approved by the FDA. The lack of clear and controlled studies has given rise to skepticism regarding this potentially very valuable approach.

In general, chelation therapy is a medical treatment that uses chelating agents to treat disorders and diseases that are induced by or result in abnormally high metal ion accumulation in the body. The basis for chelation therapy is metal-ligand bonding chemistry. The properties of the chelation agent selected are critical to the overall outcome of chelation therapy. Human evolution has defined a specific set of functions for each essential trace element in the body; abnormal concentrations or distributions of any element may result in physiological disorders or diseases. Medical interventions should ideally help to rebalance metal trafficking, rather than to perturb it. No benefit is derived from a chelating agent administered to a healthy system; on the contrary such unnecessary treatment may upset the carefully regulated system and thus be detrimental and dangerous. Chelation agents without adequate selectivity can also deplete useful elements along with toxic metals from the body, which may induce further health problems.

Over the past few decades, the advance of chelation therapy has been constrained by the intrinsic obstacles of chemical, biological and clinical restrictions; by the vague recognition of the relation between various disorders and physiological heavy metal imbalance, by inadequate interaction between chemical and biomedical researchers and by a lack of interest from the pharmaceutical industry. Above all, the lack of appropriate chelation agents and adequate clinical trials has been the bottleneck restricting the advance of chelation therapy. With the development of more metal-selective and sophisticated chelation agents such as deferoxamine (DFO), deferiprone (DFP) and deferasirox (DFX) and recent clinical trials, chelation therapy has shown great improvement over the results obtained using earlier chelation agents such as EDTA. Encouragingly, technological advances such as MRI have enabled the quantification of brain iron content, and with it the evaluation of the therapeutic effects of chelation therapy. Such advances have significantly facilitated the study of chelation therapy in this area, and promising results are being reported in a growing number.
If we regard universal ligands such as EDTA as first-generation chelation agents which lack adequate selectivity, the second generation of agents should be more selective toward specific metal ions. Furthermore, heavy metal ions in the body do not exist as free ions, but rather are typically associated with proteins or other biomolecules. This association may exert negative overall effects by interfering with normal biomolecule functions. Selective and competitive binding of the metal ions by chelation agents in the pathologic tissues may thus rescue normal function. Therefore, the requirements for next-generation chelation agents should include not only metal ion selectivity but also the ability to target certain biomolecules related to disorders and diseases. In this manner, chelation agents can be directed to where they are most needed, thereby increasing efficacy and minimizing potential off-target effects. Efforts toward this goal have already begun and have exhibited promising preliminary results. Chelation therapy is approaching a turning point that should lead to rapid progress. It is time to expedite and expand this research and to regain public confidence. With the advance of chelation therapy and the understanding of the mechanisms behind diseases induced by heavy metal overload, chelation therapy will play a more important and possibly even indispensable role in the treatments of such diseases, not simply an alternative and auxiliary treatment.

Coordination chemistry, the foundation of chelation therapy, is a well-established science; however, the gap between coordination theory and practical medical application is still wide. More fundamental research in the fields of chelation ligand development, chelation chemistry in biological environments, and ligand biodistribution and toxicity is required to solidify our understanding, and to reach our ultimate goals of clinical trials and refined therapy protocols. General studies in these areas have been reported, but journals that specifically address the advances of chelation therapy are still limited. In view of the medical merits of chelation therapy, the delayed basic research and clinical trials, and the potential for extensive and rapidly expanding application, there is enormous need to accelerate the study in this field. The inauguration of the Journal of Heavy Metal and Chelation Therapy would provide an appropriate platform to promote the advance of chelation therapy and benefit the population at large. 